1 Hacettepe Journal of Mathematics and Statistics

$\bigcap$ Volume 45 (6) (2016), $1657-1663$

\title{
Kantorovich-type operators preserving affine functions
}

\author{
Octavian Agratini*
}

\begin{abstract}
Starting from positive linear operators which have the capability to reproduce affine functions, we design integral operators of Kantorovichtype which enjoy by the same property. We focus to show that the error of approximation can be smaller than in classical Kantorovich construction on some subintervals of its domain. Special cases are presented.
\end{abstract}

Keywords: Szász-Mirakjan operator, Baskakov operator, Stancu operator, Kantorovich operator, modulus of continuity.

2000 AMS Classification: 41A36.

Received : 11.02.2015 Accepted : 19.02.2016 Doi : 10.15672/HJMS.20164515994

\section{Introduction}

In the field of Approximation Theory the study of positive and linear approximation processes holds an important place. In time numerous such sequences of discrete type operators have been investigated. In the sequel we generically denote a such sequence by $\left(L_{n}\right)_{n \geq 1}$. Among them, a special attention has been paid to operators which reproduce affine functions, property implied by the following two relations $L_{n} e_{0}=e_{0}$ and $L_{n} e_{1}=e_{1}$, $n \in \mathbb{N}$. Set $e_{j}, j \in \mathbb{N}_{0}=\{0\} \cup \mathbb{N}$, the monomial of degree $j$. Since discrete operators are not suitable for approximating discontinuous functions, they were generalized into operators of integral type. One of the usual techniques is known as Kantorovich method which leads to an approximation process, say $\left(\widetilde{L}_{n}\right)_{n \geq 1}$, in spaces of integrable functions. Usually, the integral operators keep the property to reproduce constants, this means $\widetilde{L}_{n} e_{0}=e_{0}$ but lose the property to reproduce affine functions, in other words $\widetilde{L}_{n} e_{1} \neq e_{1}$.

The primary model for such construction is given by Bernstein operators defined as follows

$$
\left(B_{n} f\right)(x)=\sum_{k=0}^{n} p_{n, k}(x) f\left(\frac{k}{n}\right), f \in \mathbb{R}^{[0,1]}, x \in[0,1],
$$

\footnotetext{
*Babeş-Bolyai University Faculty of Mathematics and Computer Science Str. Kogălniceanu, 1400084 Cluj-Napoca, Romania

Email : agratini@math.ubbcluj.ro
} 
and $p_{n, k}(x)=\left(\begin{array}{l}n \\ k\end{array}\right) x^{k}(1-x)^{n-k}, k=\overline{0, n}$. In the above $\mathbb{R}^{[0,1]}$ represents the space of all real-valued functions defined on the compact interval $[0,1]$. Kantorovich extension has the form

$$
\left(\widetilde{B}_{n} f\right)(x)=(n+1) \sum_{k=0}^{n} p_{n, k}(x) \int_{k /(n+1)}^{(k+1) /(n+1)} f(t) d t, x \in[0,1],
$$

where $f \in L_{1}([0,1])$, the space of all Lebesgue integrable functions on $[0,1]$.

One has $B_{n} e_{0}=e_{0}, B_{n} e_{1}=e_{1}, \widetilde{B}_{n} e_{0}=e_{0}$ and $\widetilde{B}_{n} e_{1} \neq e_{1}$.

The purpose of this article is the following. Starting from a general discrete linear positive process reproducing polynomials of first degree, we indicate a technique to create an integral generalization in Kantorovich sense which will inherit the same property to reproduce affine functions. We study the error of approximation of the new sequence establishing the condition in which they are more useful than classical Kantorovich-type operators. Finally we present some particular examples.

\section{The operators}

Throughout the paper we consider an interval $J \subseteq \mathbb{R}$, which may be one of the types $J=[0,1]$ or $J=\mathbb{R}_{+}=[0, \infty)$. The second variant will exhibit the problems caused by a finite endpoint and by the boundlessness of the interval. Let $\left(x_{n, k}\right)_{k \in I_{n}}$ be a net on the interval $J$, where $I_{n} \subseteq \mathbb{N}$ is a set of indices. In what follows we consider that the net has equidistant nodes, meaning that for each $n \in \mathbb{N}$,

$$
x_{n, k+1}-x_{n, k}=p_{n}, k \in I_{n},
$$

where $\lim p_{n}=0$. In fact, the overwhelming majority of discrete linear positive operators have this property. Most frequently encountered case is described by $x_{n, k}=k / n$, this implying $p_{n}=1 / n$.

We consider a sequence of linear positive operators of discrete type defined as follows

$$
\left(L_{n} f\right)(x)=\sum_{k \in I_{n}} \lambda_{n, k}(x) f\left(x_{n, k}\right), x \in J,
$$

where $\lambda_{n, k} \in C(J), \lambda_{n, k} \geq 0$ for each $(n, k) \in \mathbb{N} \times I_{n}$. For our purposes, if $\operatorname{Card}\left(I_{n}\right)$ is finite, then $f \in C(J)$. If $\operatorname{Card}\left(I_{n}\right)$ is non-finite, then

$$
f \in \mathcal{F}(J):=\{g \in C(J) \text { : the series in (2.2) is absolutely convergent }\} .
$$

Denoting by $C_{B}(J)$ the space of all real-valued continuous and bounded functions on $J$, we get $C_{B}(J) \subset \mathcal{F}(J)$. Anyway, we keep the assumption that $e_{j} \in \mathcal{F}(J), j=1$ and $j=2$. As announced in Introduction, we consider that these operators reproduce affine functions, i.e.,

$$
\sum_{k \in I_{n}} \lambda_{n, k}(x)=1
$$

and

$$
\sum_{k \in I_{n}} \lambda_{n, k}(x) x_{n, k}=x, x \in J
$$

Set $p^{*}=\sup _{n \in \mathbb{N}} p_{n}$ where $p_{n}$ is given at (2.1). If $J=\mathbb{R}_{+}$, then we consider $J^{*}=\left[\frac{p^{*}}{2}, \infty\right)$. If $J=[0,1]$, we take $J^{*}=\left[\frac{p^{*}}{2}, 1\right]$. 
We define an integral generalization in Kantorovich sense of $L_{n}, n \in \mathbb{N}$, operators as follows

$$
\left(\widetilde{L}_{n} f\right)(x)=\frac{1}{p_{n}} \sum_{k \in I_{n}} \lambda_{n, k}\left(x-\frac{p_{n}}{2}\right) \int_{x_{n, k}}^{x_{n, k+1}} f(t) d t, x \in J^{*} .
$$

If $I_{n}$ is finite, the function $f$ must be chosen integrable on $J$. Otherwise, $f$ must be locally integrable function on $J$ such that the antiderivative of $f$ to belong to the space $\mathcal{F}(J)$. Also we mention that for a certain $k \in I_{n}$ such that $x_{n, k} \in J$ and $x_{n, k+1} \notin J$ we will replace $x_{n, k+1}$ with $x_{n, k}$. In other words, the integral will become null. This can happen if $J$ is bounded.

Clearly, $\widetilde{L}_{n}, n \in \mathbb{N}$, are linear and positive operators.

The aim of this note is to show that our modified operators preserve affine functions.

In our opinion, the study of the convergence for $\left(\widetilde{L}_{n}\right)_{n \geq 1}$ sequence does not bring too much novelties. We will turn our attention to another direction. We determine in what circumstances this class of operators can offer a smaller approximation error than the classical Kantorovich operators. Some special cases are delivered.

\section{The usefulness of $\widetilde{L}_{n}$ operator}

At the beginning we calculate the first three moments of our integral operators.

Theorem 1. Let $\widetilde{L}_{n}, n \in \mathbb{N}$, be defined by (2.5). For each $x \in J^{*}$ we have

(i) $\left(\widetilde{L}_{n} e_{0}\right)(x)=1$,

(ii) $\left(\widetilde{L}_{n} e_{1}\right)(x)=x$,

(iii) $\left(\widetilde{L}_{n} e_{2}\right)(x)=\left(L_{n} e_{2}\right)\left(x-\frac{p_{n}}{2}\right)+p_{n} x-\frac{p_{n}^{2}}{6}$,

where $L_{n}$ and $p_{n}$ are defined by (2.2) and (2.1), respectively.

Proof. The first statement is a direct consequence of (2.3). By using (2.1) and (2.4) we can write

$$
\begin{aligned}
\left(\widetilde{L}_{n} e_{1}\right)(x) & =\frac{1}{2 p_{n}} \sum_{k \in I_{n}} \lambda_{n, k}\left(x-\frac{p_{n}}{2}\right)\left(2 p_{n} x_{n, k}+p_{n}^{2}\right) \\
& =\left(L_{n} e_{1}\right)\left(x-\frac{p_{n}}{2}\right)+\frac{p_{n}}{2}\left(L_{n} e_{0}\right)\left(x-\frac{p_{n}}{2}\right)=x .
\end{aligned}
$$

Similarly, by using (2.1) and (2.5), we get

$$
\left(\widetilde{L}_{n} e_{2}\right)(x)=\frac{1}{3 p_{n}} \sum_{k \in I_{n}} \lambda_{n, k}\left(x-\frac{p_{n}}{2}\right)\left(3 x_{n, k}^{2} p_{n}+3 x_{n, k} p_{n}^{2}+p_{n}^{3}\right)
$$

which leads us to the last statement of Theorem 1.

The first two identities of Theorem 1 guarantee that the operators $\widetilde{L}_{n}, n \in \mathbb{N}$, inherit property to reproduce affine functions. is

At this point we introduce the second order central moment of the operator $\widetilde{L}_{n}$, that

$$
\mu_{n, 2}(x):=\left(\widetilde{L}_{n} \varphi_{x}^{2}\right)(x), \text { where } \varphi_{x}(t)=t-x,(t, x) \in J \times J^{*} .
$$

In view of Theorem 1 , for any $x \in J^{*}$ we get

$$
\mu_{n, 2}(x)=\left(L_{n} e_{2}\right)\left(x-\frac{p_{n}}{2}\right)+x\left(p_{n}-x\right)-\frac{p_{n}^{2}}{6} .
$$

Starting from the same discrete operator $L_{n}, n \in \mathbb{N}$, it is well known that the classical Kantorovich generalization is designed as follows

$$
\left(L_{n}^{*} f\right)(x)=\frac{1}{p_{n}} \sum_{k \in I_{n}} \lambda_{n, k}(x) \int_{x_{n, k}}^{x_{n, k+1}} f(t) d t, x \in J .
$$


Following a similar path set for $\widetilde{L}_{n}, n \in \mathbb{N}$, we easily deduce the formulas for each $x \in J$.

$$
\begin{aligned}
& \left(L_{n}^{*} e_{0}\right)(x)=1, \\
& \left(L_{n}^{*} e_{1}\right)(x)=x+\frac{p_{n}}{2}, \\
& \left(L_{n}^{*} e_{2}\right)(x)=\left(L_{n} e_{2}\right)(x)+p_{n} x+\frac{p_{n}^{2}}{3} .
\end{aligned}
$$

The second order central moment is given by

$$
\mu_{n, 2}^{*}(x):=\left(L_{n}^{*} \varphi_{x}^{2}\right)(x)=\left(L_{n} e_{2}\right)(x)-x^{2}+\frac{p_{n}^{2}}{3} .
$$

We turn our attention in comparing the approximation errors caused by the two classes of operators, $\left(\widetilde{L}_{n}\right)_{n \geq 1}$ and $\left(L_{n}^{*}\right)_{n \geq 1}$. To achieve it, we recall the notion of the first modulus of smoothness associated to a continuous function $f$ on a compact interval $[a, b]$. It is denoted by $\omega(f ; \cdot)_{[a, b]}$ and is defined as follows

$$
\omega(f ; \delta)_{[a, b]}=\sup \{|f(t)-f(x)|:|t-x| \leq \delta, t, x \in[a, b]\}, \delta \geq 0 .
$$

Theorem 2. (i) Let $J=[0,1]$ and $J^{*}=\left[\frac{p^{*}}{2}, 1\right]$. For any function $f \in C(J)$, the operators $\widetilde{L}_{n}$ and $\widetilde{L}_{n}^{*}, n \in \mathbb{N}$, satisfy

$$
\left|\left(L_{n}^{*} f\right)(x)-f(x)\right| \leq 2 \omega_{J}\left(f ; \sqrt{\mu_{n, 2}^{*}(x)}\right)
$$

and

$$
\left|\left(\widetilde{L}_{n} f\right)(x)-f(x)\right| \leq 2 \omega_{J^{*}}\left(f ; \sqrt{\mu_{n, 2}(x)}\right) .
$$

(ii) Let $J=[0, \infty)$ and $J^{*}=\left[\frac{p^{*}}{2}, \infty\right)$. Let $\tau$ be fixed, $\tau>p^{*} / 2$. For any function $f \in C_{B}(J)$, the operators $\widetilde{L}_{n}$ and $L_{n}^{*}, n \in \mathbb{N}$, satisfy

$$
\left|\left(L_{n}^{*} f\right)(x)-f(x)\right| \leq 2 \omega_{[0, \tau]}\left(f ; \sqrt{\mu_{n, 2}^{*}(x)}\right)
$$

and

$$
\left|\left(\widetilde{L}_{n} f\right)(x)-f(x)\right| \leq 2 \omega_{\left[p^{*} / 2, \tau\right]}\left(f ; \sqrt{\mu_{n, 2}(x)}\right) .
$$

Proof. These quantitative results given in terms of the modulus of smoothness are direct consequence of the following statement proved by Shisha and Mond [6]. If $\Lambda$ is a linear positive operator defined on $C([a, b])$, then one has

$$
\begin{aligned}
|(\Lambda f)(x)-f(x)| & \leq|f(x)|\left|\left(\Lambda e_{0}\right)(x)-1\right| \\
& +\left(\left(\Lambda e_{0}\right)(x)+\frac{1}{\lambda} \sqrt{\left(\Lambda e_{0}\right)(x)\left(\Lambda \varphi_{x}^{2}\right)(x)}\right) \omega(f ; \lambda),
\end{aligned}
$$

for every $x \in[a, b]$ and $\lambda>0$. Taking in view that our operators $L_{n}^{*}$ and $\widetilde{L}_{n}$ reproduce the constants and choosing $\lambda=\sqrt{\left(\Lambda \varphi_{x}^{2}\right)(x)}$ the conclusions of our theorem are taken place.

Examining the upper bound of the approximation error, it is noticed that the operators $\widetilde{L}_{n}, n \in \mathbb{N}$, prove their usefulness if $\mu_{n, 2}<\mu_{n, 2}^{*}$ holds.

Theorem 3. The operators $\widetilde{L}_{n}, n \in \mathbb{N}$, defined by (2.5) as compared to operators $L_{n}^{*}, n \in \mathbb{N}$, defined by (3.2) give a better error estimation for continuous and bounded functions if

$$
\left(L_{n} e_{2}\right)(x)\left(x-\frac{p_{n}}{2}\right)-\left(L_{n} e_{2}\right)(x)+p_{n} x<\frac{p_{n}^{2}}{2}, x \geq \frac{p^{*}}{2},
$$

holds. The operators $L_{n}, n \in \mathbb{N}$, are defined by (2.2). 
Proof. Imposing $\mu_{n, 2}<\mu_{n, 2}^{*}$ and using relations (3.1) and (3.3), the conclusion follows.

\section{Examples}

The first two examples pertain to the case $J=\mathbb{R}_{+}$and in the last example we consider $J=[0,1]$.

1. Modified Szász-Mirakjan-Kantorovich operators

The classical Szász-Mirakjan operators are defined by

$$
\left(L_{n} f\right)(x)=\sum_{k=0}^{\infty} s_{n, k}(x) f\left(\frac{k}{n}\right), \text { where } s_{n, k}(x)=e^{-n x} \frac{(n x)^{k}}{k !}, x \geq 0 .
$$

The Szász-Kantorovich operators have been defined (see Butzer [1]) by

$$
\left(L_{n}^{*} f\right)(x)=n \sum_{k=0}^{\infty} s_{n, k}(x) \int_{k / n}^{(k+1) / n} f(t) d t, x \geq 0 .
$$

In this case, $p_{n}=1 / n$ and our operators $\widetilde{L}_{n}, n \in \mathbb{N}$, are given by

$$
\left(\widetilde{L}_{n} f\right)(x)=n e^{\frac{1-2 n x}{2}} \sum_{k=0}^{\infty} \frac{(2 n x-1)^{k}}{2^{k} k !} \int_{k / n}^{(k+1) / n} f(t) d t, x \geq \frac{1}{2} .
$$

These operators have been defined and studied by Duman, Özarslan and Della Vecchia [3]. The genuine Szász-Mirakjan operators satisfy

$$
\left(L_{n} e_{2}\right)(x)=x^{2}+\frac{x}{n}
$$

and substituting in $(3.4)$ we get $-3 /\left(4 n^{2}\right)<0$. This fact guarantees that $\widetilde{L}_{n}$ operators generate a smaller approximation error.

2. Modified Baskakov-Kantorovich operators

The Baskakov operators are defined by

$$
\left(L_{n} f\right)(x)=\sum_{k=0}^{\infty} v_{n, k}(x) f\left(\frac{k}{n}\right)
$$

where

$$
v_{n, k}(x)=\left(\begin{array}{c}
n+k-1 \\
k
\end{array}\right) x^{k}(1+x)^{-n-k}, x \geq 0 .
$$

As mentioned in [2, p. 115], their Kantorovich extension has the form

$$
\left(L_{n}^{*} f\right)(x)=n \sum_{k=0}^{\infty} v_{n, k}(x) \int_{k / n}^{(k+1) / n} f(t) d t .
$$

We deduce $p_{n}=1 / n$ and $\widetilde{L}_{n}$ operators are defined by

$$
\left(\widetilde{L}_{n} f\right)(x)=2^{n} n^{n+1} \sum_{k=0}^{\infty}(2 n x-1)^{k}(2 n x+2 n-1)^{-n-k} \int_{k / n}^{(k+1) / n} f(t) d t,
$$

$x \geq \frac{1}{2}$. Since

$$
\left(L_{n} e_{2}\right)(x)=x^{2}+\frac{x(1+x)}{n}
$$

relation (3.4) becomes

$$
1-3 n<4 n x, x \geq \frac{1}{2},
$$


which implies a better error approximation by using $\widetilde{L}_{n}, n \in \mathbb{N}$, operators.

3. Modified Stancu-Kantorovich operators

Stancu polynomials [7] depend on parameter $\alpha \geq 0$ and are defined by

$$
\left(L_{n} f\right)(x)=\sum_{k=0}^{n} \omega_{n, k}^{\langle\alpha\rangle}(x) f\left(\frac{k}{n}\right), x \in[0,1],
$$

where

$$
\begin{aligned}
\omega_{n, k}^{\langle\alpha\rangle}(x) & =\left(\begin{array}{l}
n \\
k
\end{array}\right) \frac{x^{(k,-\alpha)}(1-x)^{(n-k,-\alpha)}}{1^{(n,-\alpha)}}, \\
x^{(k,-\alpha)} & :=x(x+\alpha) \ldots(x+(k-1) \alpha) .
\end{aligned}
$$

The following identities

$$
L_{n} e_{0}=e_{0}, \quad L_{n} e_{1}=e_{1}, \quad L_{n} e_{2}=e_{2}+\frac{1+n \alpha}{n(1+\alpha)}\left(e_{1}-e_{2}\right)
$$

hold. The integral extension in Kantorovich sense of Stancu operators has been introduced and studied in [5]

$$
\left(L_{n}^{*} f\right)(x)=(n+1) \sum_{k=0}^{n} \omega_{n, k}^{\langle\alpha\rangle}(x) \int_{k /(n+1)}^{(k+1) /(n+1)} f(t) d t .
$$

For operators generated by formula $(2.5)$ we identify $p_{n}=1 /(n+1), p^{*}=1 / 2$ and $J^{*}=\left[\frac{1}{4}, 1\right]$. Relation $(3.4)$ becomes

$$
-1-\beta_{n}+2(n+1) \beta_{n}(2 x-1)<0,
$$

where $\beta_{n}=\frac{1+n \alpha}{n(1+\alpha)}$.

For special case $\alpha=0$, Stancu operators turn into Bernstein operators and $L_{n}^{*}$ operators become the genuine Kantorovich operators [4], see (1.1) and (1.2). In this case $\beta_{n}=1 / n$ and for each $x<3 / 4$ relation (4.1) hold. Consequently, at least on the interval $[1 / 4,3 / 4]$ the operators $\widetilde{L}_{n}, n \in \mathbb{N}$, give a better error approximation.

For $\alpha>0$, we set

$$
\tau=\inf _{n \geq 1} \frac{2 n \beta_{n}+3 \beta_{n}+1}{4(n+1) \beta_{n}} .
$$

We can easily verify that $1 / 2<\tau<1$.

In this case the interval $I$ for which the operators $\widetilde{L}_{n}, n \in \mathbb{N}$, give a better error of approximation than the operators $L_{n}^{*}, n \in \mathbb{N}$, is the following $I=\left[\frac{1}{4}, \tau\right]$.

Acknowledgment. The author thanks to the reviewer for careful reading of the manuscript which led the removal of some errors and misprints occurred in the original variant of it.

\section{References}

[1] Butzer, P.L., On the extensions of Bernstein polynomials to the infinite interval, Proc. Amer. Math. Soc., 5(1954), 547-553.

[2] Ditzian, Z., Totik, V., Moduli of Smoothness, Springer-Verlag, New York Inc., 1987.

[3] Duman, O., Özarslan, M.A., Della Vecchia, B., Modified Szász-Mirakjan-Kantorovich operators preserving linear functions, Turk. J. Math., 33(2009), 151-158.

[4] Kantorovich, L.V., Sur certains développement suivant les polynômes de la forme de S. Bernstein, I, II, C.R. Acad. URSS (1930), 563-568, 595-600.

[5] Razi, Q., Approximation of a function by Kantorovich-type operators, Matematički Vesnik, 41(1989), 183-192. 
[6] Shisa, O., Mond, B., The degree of convergence of linear positive operators, Proc. Nat. Acad. Sci. USA, 60(1968), 1196-1200.

[7] Stancu, D.D., Approximation of functions by a new class of linear polynomial operators, Rev. Roumanie Math. Pures Appl., 8(1968), 1173-1194. 
